\title{
2D Evaluation of the Potential Difference in an InP Device by Shadow Image Distortion Method
}

Katsuhiro Sasaki ${ }^{1}$ and Hirokazu Sasaki ${ }^{2}$

${ }^{1}$ UACJ Coporation, Nagoya, Aichi, Japan, ${ }^{2}$ Furukawa Electric Co. Ltd., Yokohama, Kanagawa, Japan

The shape of $\mathrm{p}$-n junction is an important information in order to evaluate the semiconductor devices, which has been observed by electron Lorentz microscopy [1], holography [2] and differential phase contrast method [3] using dedicated equipment in a limited condition. Alternative quick method is required as an industrial point of view. 2D distribution of $\mathrm{p}-\mathrm{n}$ junction in an InP laser device is evaluated by using Shadow Image Distortion (SID) method [4, 5] in a conventional transmission electron microscope (TEM) equipped with a thermal emission gun. As the results of obtained pair of images, not only the shape but also the vector of interface potential difference of $p-n$ junction has been evaluated.

The cross-sectional TEM sample of the InP laser device with the thickness in $300 \mathrm{~nm}$ is prepared by using the identical method reported in the previous work [6]. TEM observation has been performed in JEOL JEM-2100plus with using a low magnification mode imaged by objective mini-lens and a customized projector lens condition which gives the higher magnification than the standard setting. SID method visualized local potential difference as the contrast deviation in the bundle of blurred contrast of the aperture edge. The distorted shadow images are detected placing the shadow of the selected area diffraction (SAD) aperture in the image. In our previous work [5], one single image is obtained along the almost liner shadow of the largest aperture edge placed parallel to the liner interface to measure the potential difference perpendicular to the interface. In this work, two images are obtained in the identical area by placing the shadow edge of the SAD aperture along and perpendicular to the specimen edge to detect the different part of the vector of the potential difference.

In order to explain the device structure, the electron hologram in which $\mathrm{p}$ and $\mathrm{n}$ regions and the gate electrode are marked is shown in Fig. 1. Three almost liner p-n junction indicated as (1), (2) and (3), and the arc like shaped interface indicated as (4) running from the edge of strong contrast of the gate electrode are observed.

The shadow images were obtained by placing the aperture shadow edge horizontally and vertically as shown in Fig. 2a and b, respectively. As discussed in our previous work [5], SID method detects the electron beam deflection due the electrostatic field formed on the interface as the result of potential difference, at which the electron beam deflected from $\mathrm{n}$ to $\mathrm{p}$ region. When the electron beam deflected to the darker region in the image, the dark line is formed and to brighter region, the bright line is formed, respectively.

In Fig. 2a, SAD aperture hole is placed in the lower side of the image, then the shadow of the aperture plate is placed upper side of the image. On the p-n junction (1) in Fig.1 running almost horizontal direction, which deflect the electron beam to the upper direction in the image, namely, to the darker region of the image, the shallow dark line is observed in Fig. 2a. In the middle of the image, the junction (2) running in the upward right direction, which deflect the electron beam to the lower right direction, namely, to the brighter region, is observed as the bright line. In the bottom center area of the image, the near vertical junction (3) shown in Fig. 1, which deflects the electron beam to the left side of the image, namely, along the region of same brightness is not imaged. 
In Fig. 2b, SAD aperture hole is placed in the right side of the image. As the results, the contrast due to the beam deflection along the vertical direction can not be visualized. Therefore, the junction (1) imaged as shallow dark line in Fig. 2 a is not imaged, however, the junction (2) is visualized as the white line, on which the electron beam is deflected to the lower right direction, i.e., to the brighter direction in the image as mentioned above. Contrary, the junction (3) on which the electron beam is deflected to the left side of the image showed dark line. The difference of the interface contrast corresponds to the direction of the electron beam deflection.

The correspondence of the contrast of the junctions to deflection direction can be apparently found along lower part of the arc shape junction (4) The corresponding interface which runs in the upward left direction deflects the electron beam to right upper part of the image. As the result, dark line in Fig. 2a and bright line in Fig. $2 b$ as indicated thick arrows are formed, respectively. This contrast reversal on the $p$-n junction in Fig. 2a and b shows that these two images can identify the deflection vector of the electron beam on the interfaces.

When the absolute values of the deflection angle can be determined as in our previous work [5] in the horizontal (x) and vertical (y) direction, two-dimensional vectors of the projected electric field, namely, potential difference, could be determined. The obtained images in this work is very shallow and containing the contrast not only due to the electric field on the p-n junction but also the absorption contrast due to the difference of the material, for example, around the gate electrode. The next step of this work is the removal of the additional contrast effect on the image [7] by comparing with the ordinary bright field image. The spatial resolution of the image is not enough to get clear evaluation of the device in this work, however, the current limitation is due to the pixel size of CCD detector or the magnification of the projector lens system, which can be overcome improving the lens condition. As shown in Fig.1, electron hologram can already image the device structure with the dedicated equipment, which is not so suitable for industrial application, however, SID method, we think, using conventional TEM without any modification is simple enough to be applicable for the industrial application.

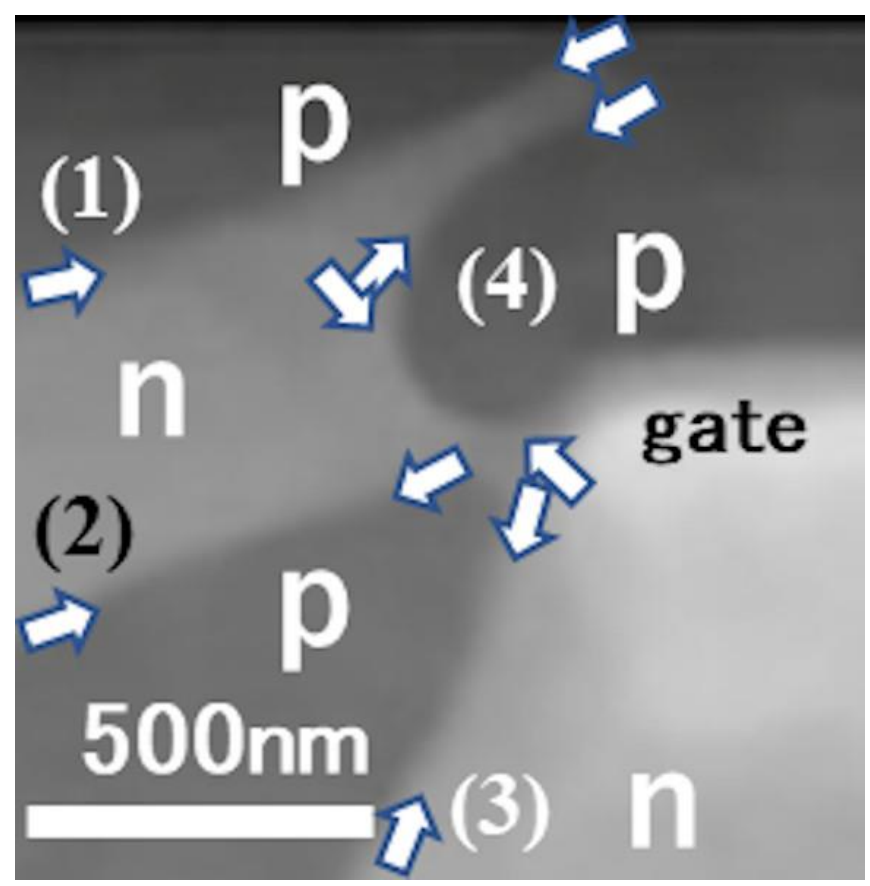

Figure 1. The electron hologram showing the device structure. 


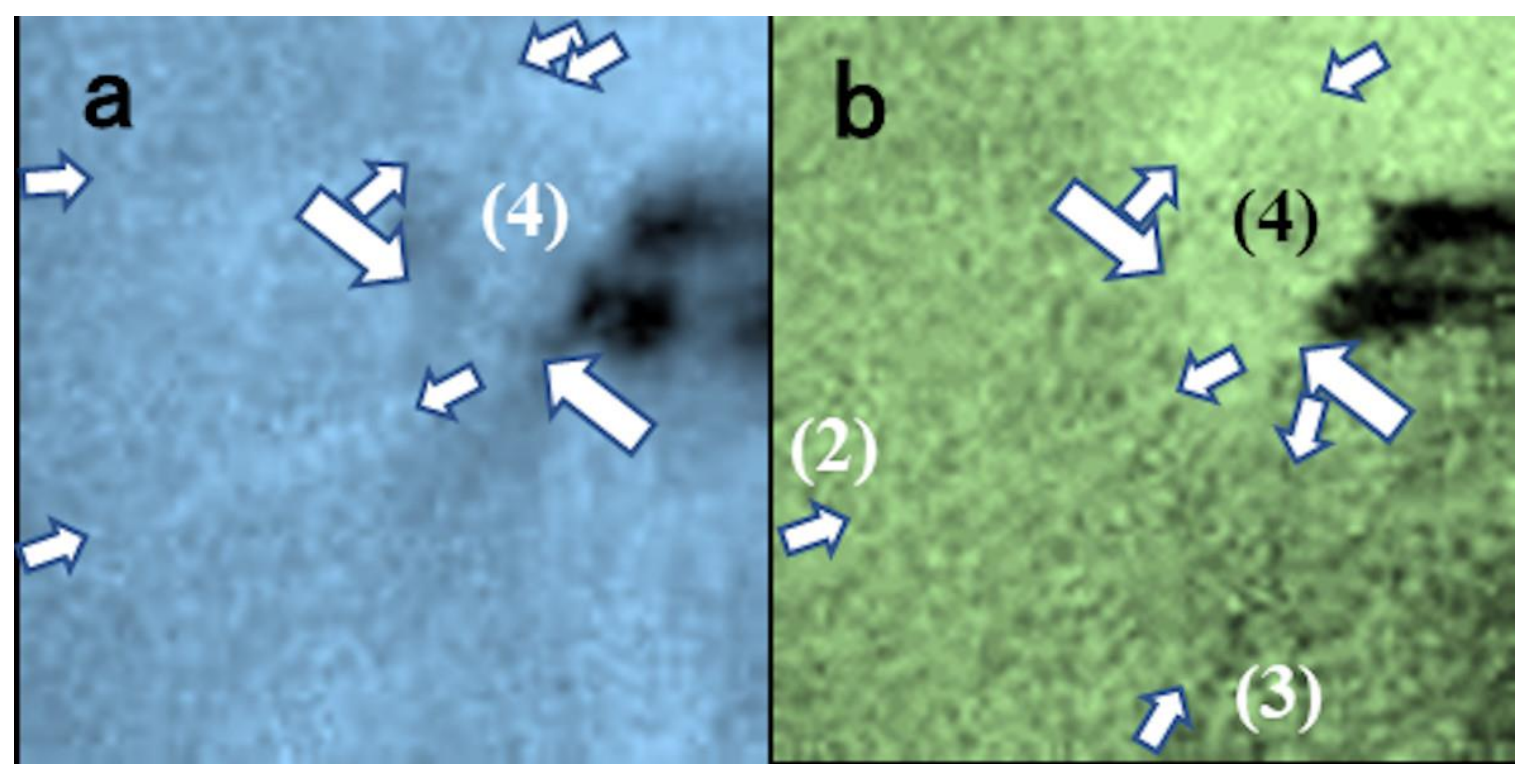

Figure 2. The shadow images placing the aperture edge (a) horizontally and (b) vertically.

\section{References}

[1] J. N. Chapman, J. Phys. D, 17 (1984) p. 623.

[2] A. Tonomura, Reviews of Modern Physics, 59 (1987) p. 639.

[3] N. Shabata, et al., Scientific Reports 5 (2015) 10040.

[4] K. Sasaki, et al., in "The Transmission Electron Microscope", ed. M. Khan, (InTech, Rijeka) p.1.

[5] K. Sasaki, H. Sasaki and S. Saito, Microsc. Microanal. 24 (Suppl. 1), (2018) p.26.

[6] H. Takahashi, et al., Proc. 16th Int. Microscopy Congress (2006) p.1104.

[7] K. Sasaki, et al., Microsc. Microanal. 25 (Suppl 2) (2019), p.106. 